\title{
Composite pulses for efficient excitation of half-integer quadrupolar nuclei in NMR of static and spinning solid samples
}

\author{
Diego Carnevale ${ }^{\mathrm{a}, *}$, Geoffrey Bodenhausen ${ }^{\mathrm{a}, \mathrm{b}, \mathrm{c}, \mathrm{d}}$ \\ a Institut des Sciences et Ingénierie Chimiques, Ecole Polytechnique Fédérale de Lausanne, EPFL, Batochime, 1015 Lausanne, Switzerland \\ ${ }^{\mathrm{b}}$ Département de Chimie, Ecole Normale Supérieure, 24 Rue Lhomond, 75231, Paris Cedex 05, France \\ ${ }^{\mathrm{c}}$ Université Pierre-et-Marie Curie, Paris, France \\ ${ }^{\mathrm{d}}$ CNRS, UMR 7203, Paris, France
}

\section{A R T I C L E I N F O}

\section{Article history:}

Received 14 October 2011

In final form 23 January 2012

Available online 2 February 2012

\begin{abstract}
A B S T R A C T
Composite pulses of the type $\left(\tau_{p}\right)_{x}\left(2 \tau_{p}\right)_{-x}\left(3 \tau_{p}\right)_{x}$ allow one to excite the central transition of nuclei with half-integer spin with enhanced efficiency compared to a simple $\left(\tau_{p}^{s p}\right)_{x}$ pulse. The method has been tested on solid samples containing sodium-23 $(I=3 / 2)$, aluminium-27 $(I=5 / 2)$ and scandium-45 $(I=7 / 2)$ under both static and magic-angle spinning conditions. Numerical simulations for $I=3 / 2$ indicate that the enhancement is due to a more efficient conversion of Zeeman population differences associated with the satellite transitions to the central transition.
\end{abstract}

(c) 2012 Elsevier B.V. All rights reserved.

\section{Introduction}

Sensitivity is no doubt the Achilles' heel of NMR spectroscopy. This is inevitably due to the small population differences between the Zeeman energy levels when an external magnetic field lifts their degeneracy [1]. In the solid state, the resulting weak signals may be further compromised by the dispersion of frequencies caused by anisotropic interactions [2]. This is particularly true for quadrupolar nuclei, which represent the vast majority of NMR-active spins. The quadrupolar interaction, which cannot be fully removed by magic-angle spinning (MAS) because of second-order terms, is often very large and results in a severe lack of spectral resolution and, consequently, in weak signal-to-noise ratios [3]. Several methods have been proposed to partially overcome these intrinsic limitations that affect the acquisition of NMR spectra of quadrupolar nuclei in the solid state. Most proposed approaches achieve signal enhancement of the central transition (CT) $m_{I}=$ $+1 / 2 \leftrightarrow m_{I}=-1 / 2$ of nuclei with half-integer spin $(I \geqslant 3 / 2)$ through the selective $r f$-manipulation of the populations of the Zeeman eigenstates [4-23]. This preparation step is then usually followed by a selective excitation of the CT. Clearly, if the populations of the satellite transitions (STs) $m_{I}= \pm 1 / 2 \leftrightarrow m_{I}= \pm 3 / 2$ can be driven away from their equilibrium values, an enhanced population difference across the CT can be obtained. These population-transfer techniques achieve signal enhancement of the CT via inversion or

Abbreviations: CT, central transition; ST, satellite transition; SQC, singlequantum coherence; TQC, triple-quantum coherence.

* Corresponding author.

E-mail address: diego.carnevale@epfl.ch (D. Carnevale). saturation of the STs [24]. In contrast to these earlier approaches, this Letter focuses on an excitation process that does not require any preparation step. We present a new recursive composite scheme [25] comprising non-selective 'hard' pulses of the form $\left(\tau_{p}\right)_{x}\left(2 \tau_{p}\right)_{-x}\left(3 \tau_{p}\right)_{x} \ldots\left(n \tau_{p}\right)_{ \pm x}$, where $\tau_{p}$ is the time length of the first pulse, $3 \leqslant n \leqslant 7$, and the phase of the last pulse is $+x$ or $-x$, for odd or even values of $n$, respectively. This may be regarded as an unusual form of time-dependent amplitude modulation where the modulation frequency decreases with time. A regular phase alternation $\pm x$ at intervals $\tau_{p}$ would generate sidebands at $\omega=\omega_{r f} \pm 1 /$ $\tau_{p}$, and larger intervals $n \tau_{p}$ would cause the sidebands to move closer together to $\omega=\omega_{r f} \pm 1 /\left(n \tau_{p}\right)$. Neither picture applies to our excitation scheme, where the $r f$ phase oscillates with increasing time steps. This achieves signal enhancement of the CT without preliminary manipulation of the populations of the ST. Our method achieves an efficient excitation of the CT single-quantum coherence (SQC) and minimizes ST SQC as well as spurious coherences of higher orders which necessarily occur during a single pulse. Given that the $r$-manipulations are much shorter than in previous approaches ( $\mu$ s instead of $\mathrm{ms}$ ), we refer to our excitation scheme

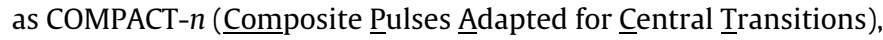
$n$ being the number of pulses.

\section{Simulations}

In order to explore the excitation profile of the various populations and coherences during a composite pulse, numerical simulations have been carried out for a quadrupolar spin $I=3 / 2$ with the SIMPSON program [26]. We make use of irreducible spherical tensor operators $T_{l, p}$ [27], of rank $l$ and coherence order 

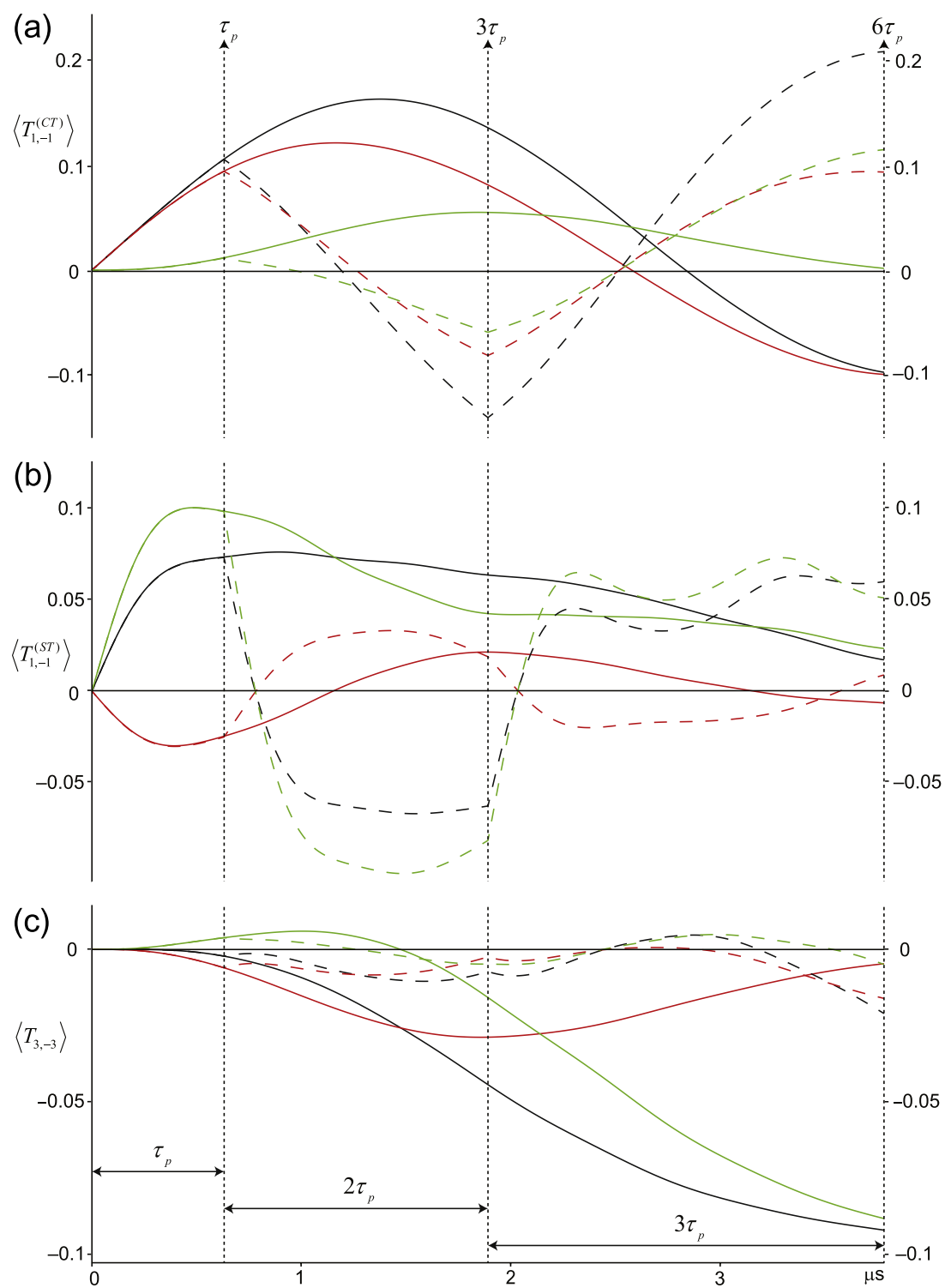

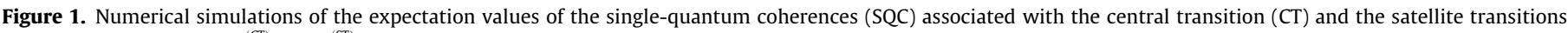

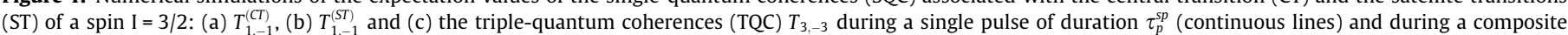

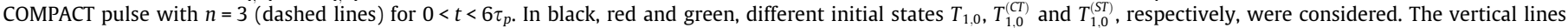

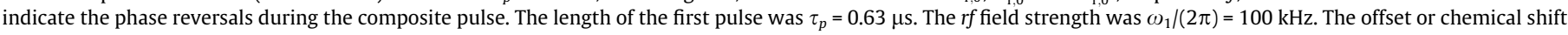

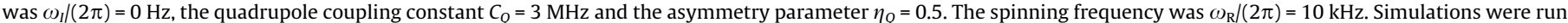
for 256 crystallite orientations and $3 \gamma$ angles.

Table 1

Expectation values of $T_{1-1}^{(C T)}, T_{1-1}^{(S T)}$ and $T_{3,-3}$ at the end of a single pulse and after a composite COMPACT-3 pulse. In the case of the single pulse, the expectation values of the three operators shown in bold indicate the maxima that are achieved at the corresponding pulse widths.

\begin{tabular}{lccc}
\hline Pulse length $(\mu \mathrm{s})$ & $\left\langle T_{1,-1}^{(C T)}\right\rangle$ & $\left\langle T_{1,-1}^{(S T)}\right\rangle$ & $\left\langle T_{3,-3}\right\rangle$ \\
\hline Single pulse & & & \\
$\tau_{p}^{s p}=0.89$ & 0.138 & $\mathbf{0 . 0 7 6}$ & -0.007 \\
$\tau_{p}^{s p}=1.39$ & $\mathbf{0 . 1 6 3}$ & 0.071 & -0.022 \\
$\tau_{p}^{s p}=3.78$ & -0.097 & 0.017 & $-\mathbf{0 . 0 9 2}$ \\
COMPACT-3 & & & \\
$6 \tau_{p}=3.78$ & $\mathbf{0 . 2 0 8}$ & 0.060 & -0.021 \\
\hline
\end{tabular}

$p$. The operator $T_{1,-1}$ corresponds to observable (in-phase) singlequantum coherence (SQC), and $T_{3,-3}$ describes triple-quantum coherence (TQC). It is convenient to decompose the single-quantum operator $T_{1,-1}$ into $T_{1,-1}=T_{1,-1}^{(C T)}+T_{1,-1}^{(S T)}$, where $T_{1,-1}^{(C T)}$ corresponds to the CT and $T_{1,-1}^{(S T)}$ to the sum of the STs. Furthermore, we decompose the Zeeman order $T_{1,0}$ into $T_{1,0}=T_{1,0}^{(C T)}+T_{1,0}^{(S T)}$ and consider these two terms separately as initial operators, so as to monitor how the populations of the Zeeman eigenstates contribute to the creation of coherences (off-diagonal elements). The following tensor operators can be detected if the signal is defined to be $s(t)=\operatorname{Tr}\left[T_{l, p} \sigma(t)\right]$ with $l=1$ and $p=-1$ :

$$
T_{1,-1}^{(C T)}=\frac{1}{\sqrt{10}}\left(\begin{array}{cccc}
0 & 0 & 0 & 0 \\
0 & 0 & 0 & 0 \\
0 & 2 & 0 & 0 \\
0 & 0 & 0 & 0
\end{array}\right), \quad T_{1,-1}^{(S T)}=\frac{1}{\sqrt{10}}\left(\begin{array}{cccc}
0 & 0 & 0 & 0 \\
\sqrt{3} & 0 & 0 & 0 \\
0 & 0 & 0 & 0 \\
0 & 0 & \sqrt{3} & 0
\end{array}\right)
$$




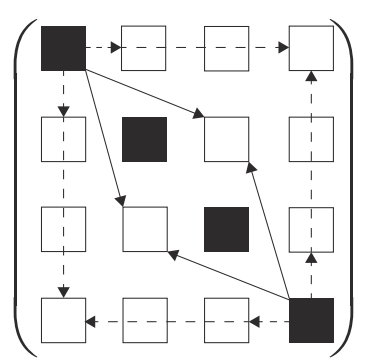

Figure 2. Schematic representation of the overall effect of the composite pulse on the density matrix. The creation of ST SQC and TQC from outer Zeeman states is quenched (dashed arrows). The outer diagonal elements are instead redirected towards the CT SQC (continuous arrows).

while the TQC with $p=-3$ has the following form

$T_{3,-3}=\left(\begin{array}{cccc}0 & 0 & 0 & 0 \\ 0 & 0 & 0 & 0 \\ 0 & 0 & 0 & 0 \\ 1 & 0 & 0 & 0\end{array}\right)$

and the initial states that are considered separately in the simulations are:

$T_{1,0}^{(C T)}=\frac{1}{\sqrt{20}}\left(\begin{array}{cccc}0 & 0 & 0 & 0 \\ 0 & 1 & 0 & 0 \\ 0 & 0 & -1 & 0 \\ 0 & 0 & 0 & 0\end{array}\right)$ and $T_{1,0}^{(S T)}=\frac{1}{\sqrt{20}}\left(\begin{array}{cccc}3 & 0 & 0 & 0 \\ 0 & 0 & 0 & 0 \\ 0 & 0 & 0 & 0 \\ 0 & 0 & 0 & -3\end{array}\right)$.

Extensions to spins $I=5 / 2,7 / 2$ and $9 / 2$ are obvious. We consider the quadrupolar interaction to second order, as implemented in Simpson. Figure $1 \mathrm{a}-\mathrm{c}$ shows the expectation values of the three operators of Eq. (1a) and (1b) during a single pulse or during our COMPACT-3 sequence $\left(\tau_{p}\right)_{x}\left(2 \tau_{p}\right)_{-x}\left(3 \tau_{p}\right)_{x}$, (continuous and dashed lines, respectively). The discontinuities in the dashed curves, highlighted by vertical lines, indicate the inversions of the $r f$ phase. Simulations starting from the initial states $T_{1,0}=T_{1,0}^{(C T)}+T_{1,0}^{(S T)}$ are shown in black, $T_{1,0}^{(C T)}$ in red, and $T_{1,0}^{(S T)}$ in green. The length of the first pulse of the COMPACT-3 sequence was $\tau_{p}=0.63 \mu \mathrm{s}$. The time dependences in the CT subspace are shown in Figure 1a. At the end of the composite pulse, i.e., at $t=6 \tau_{p}=3.78 \mu \mathrm{s}$, when starting from the full Zeeman order $T_{1,0}$ (black curves), a higher CT intensity is obtained than with a single pulse with $\tau_{p}^{s p}=1.39 \mu$ s (expectation values $\left\langle T_{1,-1}^{(C T)}\right\rangle=0.208$ and 0.163 , respectively). Considering the universal bound discussed by Sørensen [28-31] for an initial state $T_{1,0}$ of a spin $I=3 / 2$, the maximum amplitude $a_{\max }$ of the Hermitian operator $T_{1,1}+T_{1,-1}$ that can be created by a unitary transformation is $\left|a_{\max }\right|=\sqrt{2} / 5 \sim 0.283$. The composite pulse therefore achieves an excitation of single-quantum CT coherence which approaches the theoretical maximum achievable much better than a single pulse ( $\sim 74 \%$ and $\sim 58 \%$, respectively, for a spin $I=3 / 2$ with parameters of Figure 1). For comparison, a single pulse with hypothetical (unrealistic) $r f$ field strengths $\omega_{1}=1$ or $2 \mathrm{MHz}$ would yield expectation values of the CT-SQC $\left\langle T_{1,-1}^{(C T)}\right\rangle=0.260$ or 0.276 , respectively. It is worth noting that an intense $r f$ field $\omega_{1} \sim 340 \mathrm{kHz}$ would be required for a single pulse to yield the same CT-SQC $\left\langle T_{1,-1}^{(C T)}\right\rangle=0.208$ as COMPACT-3 with $\omega_{1}=100 \mathrm{kHz}$. It is interesting to note that the optimal initial pulse length $\tau_{\mathrm{p}}$ of a composite pulse with $n=3$ amounts to about half of the optimum length $\tau_{p}^{s p}$ of a single pulse, i.e., $\tau_{p} \sim \tau_{p}^{s p} / 2$. Remarkably, when the operator $T_{1,0}^{(C T)}$ is artificially considered as initial state (red curves), no enhancement is obtained at the end of the composite pulse. The results obtained with the starting state represented by $T_{1,0}^{(S T)}$ are shown in green. Surprisingly, at the end of the composite pulse the amount of $T_{1,-1}^{(C T)}$ generated from the populations of the outer $m_{I}= \pm 3 / 2$ Zeeman eigenstates is higher than that obtained from the inner $m_{I}= \pm 1 / 2$ eigenstates ( 0.115 and 0.093 , respectively). Figure $1 \mathrm{~b}$ shows analogous simulations for the detectable operator $T_{1,-1}^{(S T)}$. When the full Zeeman order is considered as starting state (black lines), the amount of satellite SQ coherence $T_{1,-1}^{(S T)}$ obtained at the end of the composite pulse is smaller than that obtained with a single pulse of duration $\tau_{p}^{s p}=1.39 \mu$ s ( 0.060 and 0.071 , respectively). Another interesting finding highlighted in Figure $1 \mathrm{~b}$ is that the outer and inner Zeeman eigenstates (red and green lines, respectively) contribute to $T_{1,-1}^{(S T)}$ with opposite signs and interfere destructively at the beginning of the excitation profile. Figure 1c shows the profile related to the TQC operator $T_{3,-3}$. By inspection of the profiles obtained with three distinct initial operators, is clear that, in the

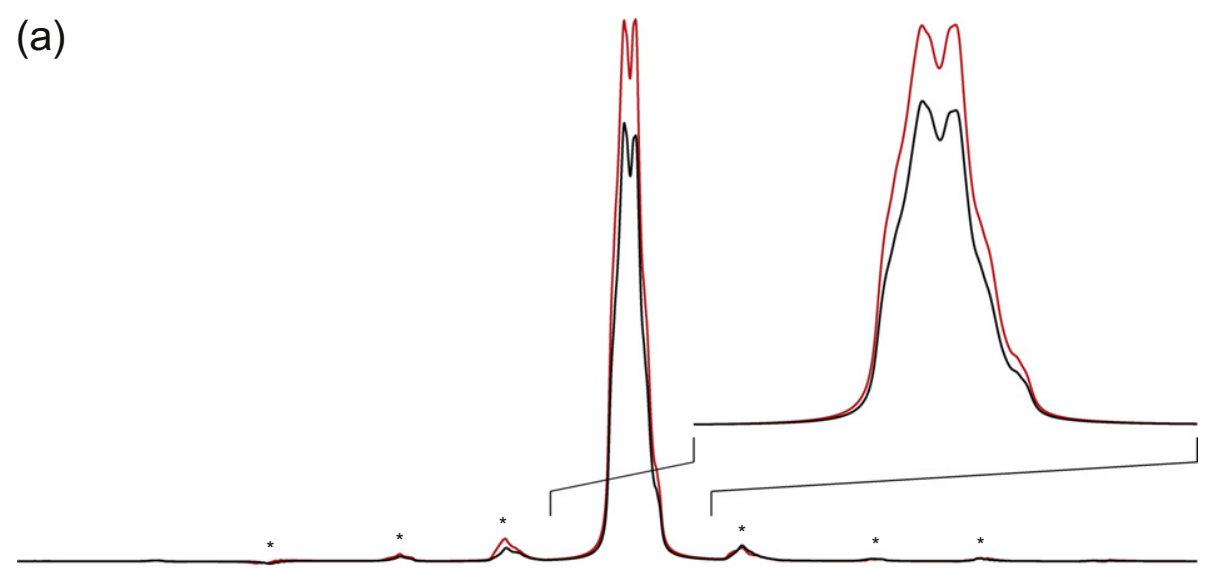

(b)

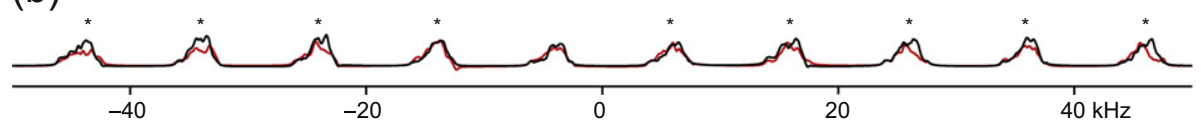

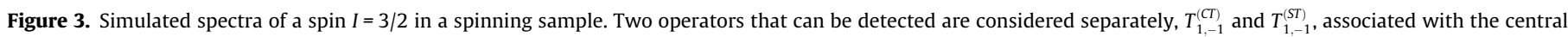

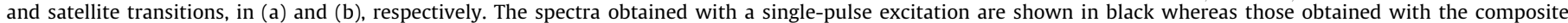

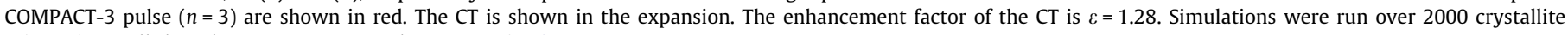
orientations. All the other parameters are the same as in Figure 1. 
single-pulse case, the TQC is generated mainly from the populations of the outer $m_{I}= \pm 3 / 2$ states. This is not surprising since the TQ transition spans these eigenstates [32]. By contrast, the action of the composite pulse causes this coherence to oscillate around zero. At the end of the COMPACT sequence, the TQC is largely quenched when compared with the case of a single pulse at the same time $6 \tau_{p}=3.78 \mu$ s $(-0.021$ and -0.092 , respectively). The expectation values of the relevant tensors created from the full Zeeman order $T_{1,0}$ are summarized in Table 1 . These simulations indicate a scenario in which the COMPACT sequence results in a quenching of the generation of ST SQC and TQC from the outer populations represented by density-matrix elements $\sigma_{1,1}$ and $\sigma_{4,4}$, and instead 'redirects' these diagonal elements towards the desired offdiagonal CT element $\sigma_{2,3}$. Figure 2 shows the overall effect of the composite excitation. Figure 3a shows, in black and red, respectively, simulations of spectra obtained with a conventional single-pulse excitation and with a COMPACT-3 pulse when the operator $T_{1,-1}^{(C T)}$ is detected. Figure $3 \mathrm{~b}$ shows the analogous spectrum obtained if the observable is the operator $T_{1,-1}^{(S T)}$. The CT is clearly enhanced by the composite pulse. The decrease of ST intensity already shown in Figure $1 \mathrm{~b}$ and Table 1 is also identified, particularly for the ST spinning-sideband envelope.

In general, we find that the optimal length $\tau_{p}$ of the first pulse of a COMPACT $n$ sequence is $\tau_{p} \sim \tau_{p}^{s p} /(n-1)$. This is highlighted in Figure $4 \mathrm{a}$, where the numerically optimized duration of the first pulse $\tau_{p}$ of composite pulses of increasing sophistication, i.e., with increasing $n$, is shown in black. In red, the function $\tau_{p}=\tau_{p}^{s p} /(n-1)$ is shown for comparison. Figure 4a shows that, as $n$ is incremented, the optimum length of the first pulse becomes progressively shorter. Clearly, this function represents a sound criterion to estimate the best length of the first pulse for a given number $n$ of pulses that make up the composite pulse. We like to stress at this point that the length of the first pulse is the only experimental parameter that needs to be optimized in this new excitation scheme, which is therefore no more demanding than a conventional single-pulse experiment. Of course, in practice, the hardware allows neither the use of infinitely short pulses, nor instantaneous phase inversions. The shortest first pulse considered in the simulations was $\tau_{p}=0.20 \mu \mathrm{s}$ for $n=9$, which is close to the technical limit of our NMR spectrometer. We found discrepancies between experimental results and simulations for $n>7$. It is worth noting that, as the length of the first pulse decreases (with increasing number of pulses $n$ ), the total length of the composite pulse increases since $\tau_{\text {tot }}=\sum_{j=1}^{n} j \tau_{p}$. Besides limitations of the amplifier, the discrepancy between experiments and simulations may be in part ascribed to homogeneous decay which alters the behavior of the coherences during the composite pulse and becomes more prominent as the overall length of the composite pulse increases. The progressive decrease of the length of the first pulse required to achieve an optimal excitation suggests that one should pay attention to the effective final flip angle $\beta_{\text {eff }}$ achieved after the $n$-th pulse. The effect of an $r f$ pulse on a half-integer quadrupolar spin has been considered by several authors in the limiting cases where $\omega_{1} \gg \omega_{Q}$ and $\omega_{1} \ll \omega_{Q}$ $[2,33,34]$. In the former case, when the quadrupolar interaction is negligible and only the external $r f$ manipulation needs to be considered, the relevant density matrix element at the end of an $x$-pulse of length $t$ is $\langle m|\sigma(t)| m+1\rangle=\frac{i}{2} \xi \sin \left(\omega_{1} t\right)$, and the signal due to a coherence associated with an arbitrary transition $m_{I} \leftrightarrow-$ $m_{I}+1$ is proportional to $\left\langle I_{y}\right\rangle=-\xi \sin \left(\omega_{1} t\right)$, where $\xi=$ $\sqrt{I(I+1)-m(m+1)}$. In the other limit, when the quadrupolar coupling is the main interaction present, the pulse can be considered to be selective, acting either on the CT or on one ST only. This allows one to recast the evaluation in a pseudo two-state $(|m\rangle,|m+1\rangle)$ basis, i.e., as if only two eigenstates were present. The relevant submatrix element at time $t$ is then $\langle m| \sigma(t)$ $|m+1\rangle=\frac{i}{2} \sin \left(\xi \omega_{1} t\right)$, and the expectation value of the detected
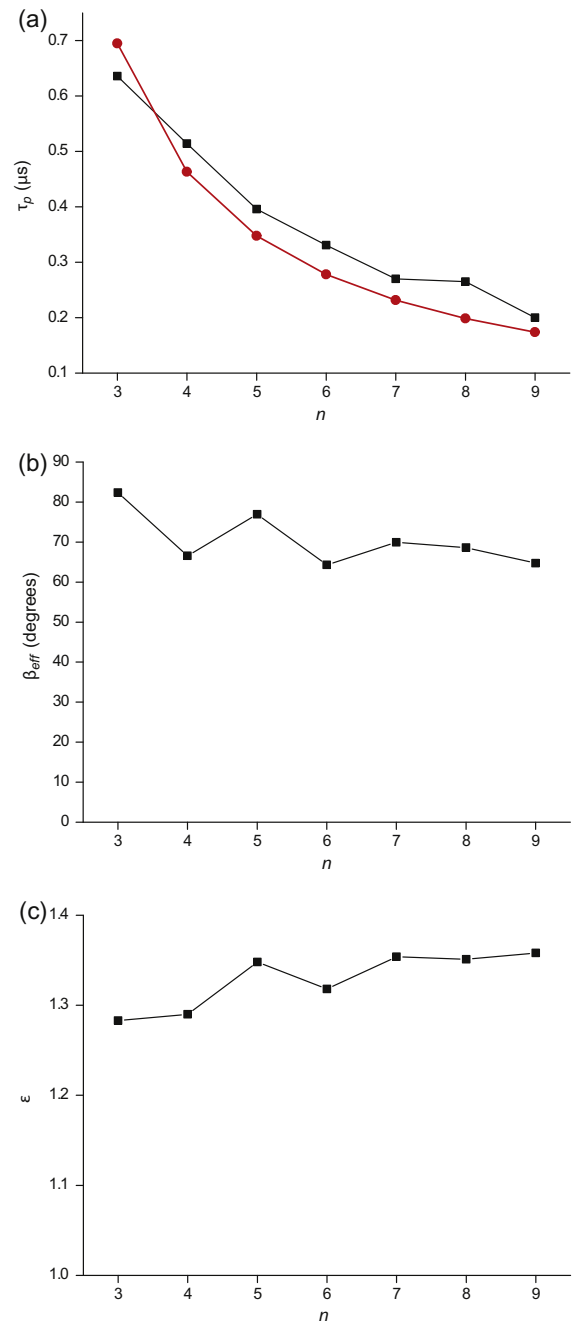

Figure 4. (a) Optimal length $\tau_{p}$ of the first pulse in a composite COMPACT- $n$ sequence versus the number of pulses $n$. In red, the function $\tau_{p}=\tau_{p}^{s p} /(n-1)$ is shown for comparison, where $\tau_{p}^{s p}$ is the optimum length of a single pulse. (b) Effective final flip angle $\beta_{\text {eff }}$ achieved at the end of the composite pulse versus the number of pulses $n$. (c) Enhancement $\varepsilon$ obtained for increasing $n$. All parameters used for the simulations were as in Figure 1.

operator in the $(|m\rangle,|m+1\rangle)$ subspace is $\left\langle I_{y}\right\rangle=-\sin \left(\xi \omega_{1} t\right)$. In this limit $\omega_{1} \ll \omega_{0}$, the CT nutates $\xi$ times faster than if the quadrupole interaction were negligible. For a spin $I=3 / 2, \xi=2$ and $\sqrt{3}$ for the CT and the ST, respectively [35-37]. In more challenging cases when $\omega_{1} \sim \omega_{Q}$, the efficiency of the excitation depends on both $\omega_{1}$ and $\omega_{0}$, the latter being orientation dependent. In the simulations of Figure 1 , we find that $\xi_{\text {eff }}=2.5 / 1.39=1.80$, which is equal to the ratio between the pulse length that would be required for a $90^{\circ}$ pulse with an $r f$ field of $100 \mathrm{kHz}$ applied to a spin $I \geqslant 3 / 2$ without quadrupolar splitting $\left(C_{Q}=0 \mathrm{MHz}\right)$, and the length of a $90^{\circ}$ pulse with the same $r f$ field strength applied to the CT of a spin system $I=3 / 2, C_{Q}=3 \mathrm{MHz}$ and $\eta=0.5$ as in our work. This factor allows one to evaluate the effective flip angle $\beta_{\text {eff }}$ achieved at the end of the COMPACT- $n$ sequence for the CT:

$\beta_{\text {eff }}=\sum_{i=1}^{n}(-1)^{i+1} i \xi_{\text {eff }} \omega_{1} \tau_{p}$

which takes into account the phase switching during the composite pulse. The numerically calculated effective final flip angles for $3 \leqslant n \leqslant 9$ are shown in Figure $4 \mathrm{~b}$. Clearly, regardless of the number of pulses considered in the composite-excitation scheme, the final 
flip angle $\beta_{\text {eff }}$ achieved with the oscillating $r f$-phase is always smaller than that which would ensure maximum intensity with a single pulse, i.e., $\beta^{s p}=\xi_{\text {eff }} \omega_{1} \tau_{p}^{s p}=90^{\circ}$, with the spin-system parameters of Figure 1. This observation is particularly interesting if one considers that, for all values of $n$ investigated, the smaller final flip angle at the end of the composite pulse always grants enhancement factors which range from $1.28<\varepsilon<1.36$. Thus, paradoxically, the COMPACT- $n$ excitation achieves a higher intensity of CT coherence with a smaller overall flip angle than that of a single pulse. Figure $4 \mathrm{~b}$ also shows that composite-pulse schemes with both odd and even $n$ tend to a common effective final nutation angle which, for the $n=9$ case, is $\beta_{\text {eff }} \sim 65^{\circ}$. Figure $4 \mathrm{c}$ shows enhancement factors calculated for the COMPACT $n$ scheme as a function of the number of pulses $n$. A smooth increase of the enhancement factor $\varepsilon$ is observed as the number of pulses $n$ is increased. In the spin system considered in the simulations, a nearly optimal enhancement seems to be obtained when $n=5$. It should be stressed at this point that earlier approaches [4-23] can grant higher enhancements than those reported in this study albeit at the cost of more complex prescriptions.

\section{Results}

The performance of the composite-pulse excitation method has been tested on powder samples of $\mathrm{Na}_{2} \mathrm{C}_{2} \mathrm{O}_{4}$ for ${ }^{23} \mathrm{Na}(I=3 / 2), \mathrm{Al}(\mathrm{a}-$ $\mathrm{Cac})_{3}$ for ${ }^{27} \mathrm{Al}(I=5 / 2)$ and $\mathrm{Sc}(\mathrm{OTf})_{3}$ for ${ }^{45} \mathrm{Sc}(I=7 / 2)$. COMPACT- $n$ excitations have been considered with $3 \leqslant n \leqslant 7$. Higher values of $n$ did not produce the enhancements expected from the simula- tions, probably indicating that the short length $\tau_{p}$ of the first pulse for $n>7$ approaches the limit of our spectrometer. All $n$ values that were experimentally explored produced very similar results. In Figure $5 \mathrm{a}$, the ${ }^{23} \mathrm{Na}$ spectrum of $\mathrm{Na}_{2} \mathrm{C}_{2} \mathrm{O}_{4}$ obtained with a single pulse was overlaid with that obtained with COMPACT- 4 excitation. An enhancement factor of $\varepsilon=1.37$ is obtained. No significant lineshape distortions were observed. The fitting of the spectra gave $C_{Q}=2.39 \mathrm{MHz}$ in both cases, and $\eta=0.71$ and 0.73 , respectively. In Figure $5 \mathrm{~b}$, the ${ }^{27} \mathrm{Al}$ spectrum of $\mathrm{Al}(\mathrm{acac})_{3}$ obtained with a single pulse was overlaid with that obtained with COMPACT-7 excitation. An enhancement factor of $\varepsilon=1.32$ was obtained. The fitting of the spectra gave $C_{Q}=2.99$ and $2.98 \mathrm{MHz}$, and $\eta=0.12$ and 0.15 , respectively. In Figure $5 c$, the ${ }^{45} \mathrm{Sc}$ spectrum of polymorphic $\mathrm{Sc}(\mathrm{OTf})_{3}$ obtained with a single pulse was overlaid with that obtained with COMPACT-4 excitation. A slightly smaller enhancement factor of $\varepsilon=1.30$ was obtained. No significant lineshape distortions were observed in this polymorphic sample. Numerical simulations show that COMPACT excitation also works on static samples. This is experimentally proven in Figure $5 d$, where the static ${ }^{23} \mathrm{Na}$ spectrum of $\mathrm{Na}_{2} \mathrm{C}_{2} \mathrm{O}_{4}$ obtained with a single pulse and that obtained with COMPACT-7 excitation were overlaid. An enhancement factor of $\varepsilon=1.34$ was obtained. The fitting of the spectra gives $C_{Q}=2.39 \mathrm{MHz}$ in both cases, and $\eta=0.85$ and 0.84 , respectively. The different asymmetry values obtained when compared to the spinning samples of Figure 5a can probably be ascribed to the removal of dipolar interactions and shielding anisotropies when MAS is utilized.
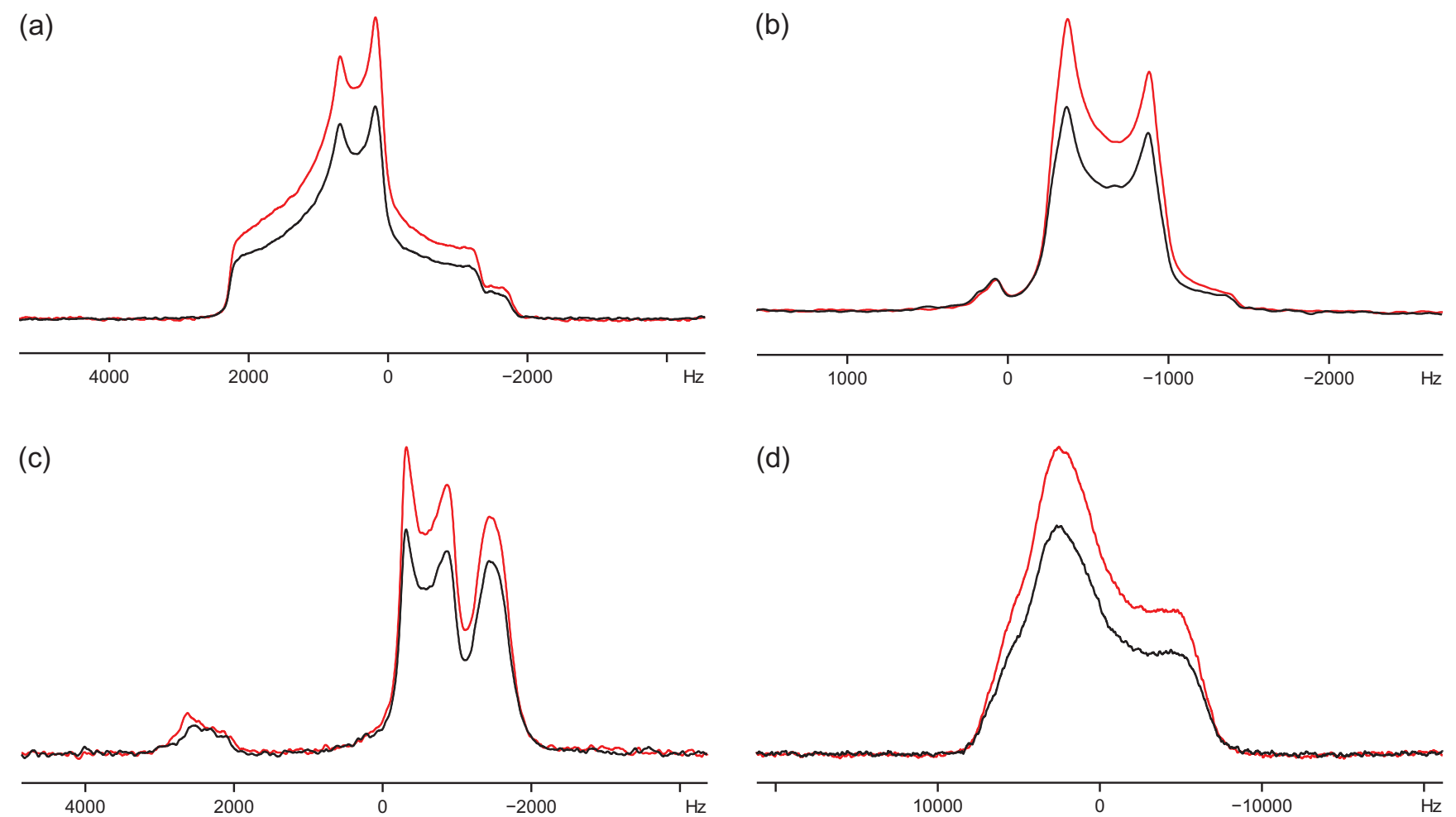

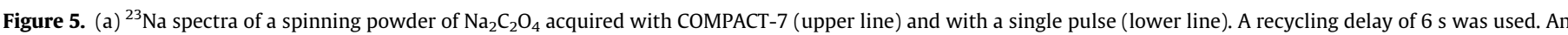

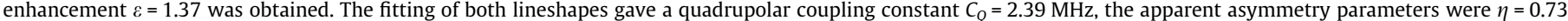

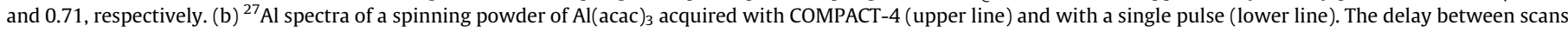

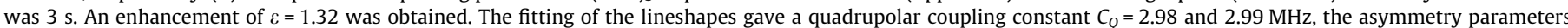

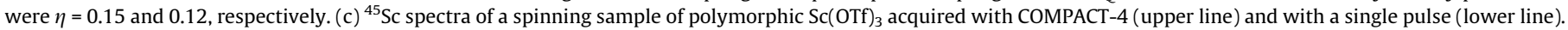

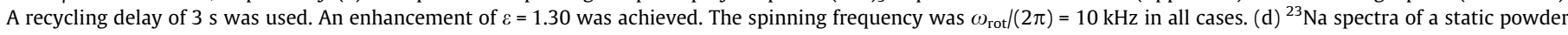

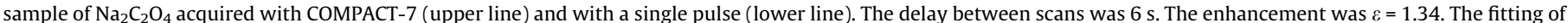

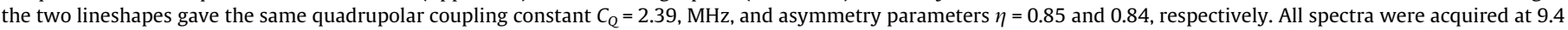
$\mathrm{T}$, and the $r f$ field strength was $\omega_{1} /(2 \pi) \sim 100 \mathrm{kHz}$ in all cases. All spectra were referenced so that the carrier frequency falls at $0 \mathrm{~Hz}$. 


\section{Conclusions}

The proposed new composite COMPACT- $n$ pulses for the excitation of the CT of half-integer quadrupolar nuclei yields enhancement factors $1.30<\varepsilon<1.4$ compared with conventional single-pulse excitation. The method has been successfully tested on samples containing $I=3 / 2,5 / 2$ and $7 / 2$. The new excitation scheme works under both static and spinning conditions. All crystallite orientations are affected in a uniform manner, resulting in unperturbed lineshapes. Numerical simulations for a spin $I=3 / 2$ indicate that the enhancement is related to a more efficient conversion into SQC of Zeeman populations associated with the ST. The ST SQC and TQC unwittingly excited from the outer eigenstates by the first pulse are partially suppressed by the phase alternation during the composite-pulse scheme. The enhancements obtained from the simulations agree remarkably well with the experimental results.

\section{Experimental and computational details}

All experiments were performed on a Bruker Avance II spectrometer equipped with a $9.4 \mathrm{~T}$ magnet $(400 \mathrm{MHz}$ for protons) and a double-channel MAS probe designed for low-temperature DNP. All measurements were performed at $300 \mathrm{~K}$. The samples were packed in $3.2 \mathrm{~mm}$ zirconia rotors and the spinning frequency was $10 \mathrm{kHz}$. The recovery delays between scans were 3 or $6 \mathrm{~s}$ and the $r f$ field strength was always $\omega_{1} /(2 \pi) \sim 100 \mathrm{kHz}$. All samples were purchased from commercial sources and used without further purification. Numerical simulations were carried out with the SIMPSon program. The quadrupolar interaction has been considered to second order. Typically 256 crystallite orientations and three $\gamma$ angles were considered, sampled with the REPULSION scheme [38].

\section{Acknowledgements}

This work was supported by the Swiss National Science Foundation, the Ecole Polytechnique Fédérale de Lausanne (EPFL), the Swiss Commission for Technology and Innovation (CTI), and the French CNRS.

\section{References}

[1] R.R. Ernst, G. Bodenhausen, A. Wokaun, Principles of Nuclear Magnetic Resonance in One and Two Dimensions, Clarendon, Oxford, 1987.

[2] M.J. Duer, Solid-State NMR Spectroscopy: Principles and Applications, Blackwell Science, UK, 2002.

[3] S.E. Ashbrook, Phys. Chem. Chem. Phys. 11 (2009) 6892.

[4] R.V. Pound, Phys. Rev. 79 (1950) 685.

[5] S. Vega, Y. Naor, J. Chem. Phys. 75 (1981) 75.

[6] J. Haase, M.S. Conradi, Chem. Phys. Lett. 209 (1993) 287.

[7] J. Haase, M.S. Conradi, C.P. Grey, A.J. Vega, J. Magn. Reson. A 109 (1994) 90.

[8] E. Van Veenendaal, B.H. Meier, A.P.M. Kentgens, J. Magn. Reson. A 109 (1998) 195.

[9] A.P.M. Kentgens, R. Verhagen, Chem. Phys. Lett. 300 (1999) 435

[10] P.K. Madhu, A. Goldbourt, L. Frydman, S. Vega, Chem. Phys. Lett. 307 (1999) 41.

[11] D. Iuga, H. Schafer, R. Verhagen, A.P.M. Kentgens, J. Magn. Reson. 147 (2000) 192.

[12] Z. Yao, H. Kwak, D. Sakellariou, L. Emsley, P.J. Grandinetti, Chem. Phys. Lett. 327 (2000) 85.

[13] D. Iuga, A.P.M. Kentgens, J. Magn. Reson. 158 (2002) 65

[14] P.K. Madhu, K.J. Pike, R. Dupree, M.H. Levitt, M.E. Smith, Chem. Phys. Lett. 367 (2003) 150.

[15] H.T. Kwak, S. Prasad, T. Clark, P.J. Grandinetti, Solid State Nucl. Magn. Reson. 24 (2003) 71.

[16] R. Siegel, T.T. Nakashima, R.E. Wasylishen, Chem. Phys. Lett. 388 (2004) 441.

[17] T. Bräuniger, K. Ramaswamy, P.K. Madhu, Chem. Phys. Lett. 383 (2004) 403.

[18] R. Siegel, T.T. Nakashima, R.E. Wasylishen, Chem. Phys. Lett. 403 (2005) 353.

[19] T. Bräuniger, G. Hempel, P.K. Madhu, J. Magn. Reson. 181 (2006) 68.

[20] R. Siegel, T.T. Nakashima, R.E. Wasylishen, Chem. Phys. Lett. 184 (2007) 85.

[21] T.T. Nakashima, R.E. Wasylishen, R. Siegel, K. Ooms, Chem. Phys. Lett. 450 (2008) 417.

[22] N.M. Trease, K.K. Dey, P.J. Grandinetti, J. Magn. Reson. 200 (2009) 334.

[23] P.K. Madhu, A. Goldbourt, L. Frydman, S. Vega, J. Chem. Phys. 112-5 (2000) 2377.

[24] R. Siegel, T.T. Nakashima, R.E. Wasylishen, Concepts Magn. Reson. 26A (2005) 47.

[25] M.H. Levitt, in: D.M. Grant, R.K. Harris (Eds.), Encyclopedia of Nuclear Magnetic Resonance, Wiley, 1996, p. 1396.

[26] M. Bak, J.T. Rasmussen, N.C. Nielsen, J. Magn. Reson. 147 (2000) 296.

[27] N. Müller, G. Bodenhausen, R.R. Ernst, J. Magn. Reson. 75 (1987) 297.

[28] O.W. Sørensen, Prog. Nucl. Magn. Reson. 21 (1989) 503.

[29] O.W. Sørensen, J. Magn. Reson. 86 (1990) 435.

[30] C.E. Hughes, S. Wimperis, J. Chem. Phys. 106 (1997) 2105

[31] C.E. Hughes, R. Kemp-Harper, S. Wimperis, J. Chem. Phys. 108 (1998) 876

[32] D. Iuga, A.P.M. Kentgens, Chem. Phys. Lett. 343 (2001) 556.

[33] D. Fenzke, D. Freude, T. Fröhlich, J. Haase, Chem. Phys. Lett. 111 (1984) 171.

[34] P.P. Man, J. Klinowski, A. Trokiner, H. Zanni, P. Papon, Chem. Phys. Lett. 151 (1988) 143.

[35] S. Vega, Phys. Rev. A 23 (1981) 3152

[36] S.E. Ashbrook, S. Wimperis, Mol. Phys. 98 (2000) 1.

[37] S.E. Ashbrook, S. Wimperis, J. Chem. Phys. 131 (2009) 194509.

[38] M. Bak, N.C. Nielsen, J. Magn. Reson. 125 (1997) 132. 